\title{
Análise espacial da distribuição dos casos de dengue e sua relação com fatores socioambientais no estado da Paraíba, Brasil, 2007-2016
}

\author{
Spatial analysis of the distribution of dengue cases and its \\ relationship with socio-environmental factors in the state of Paraiba, \\ Brazil, 2007-2016
}

Ellen Tayanne Carla da Silva', Ricardo Alves Olinda', Anna Stella Pachá1, Arthur Oliveira Costa², Alisson Lima Brito ${ }^{2}$, Dixis Figueroa Pedraza ${ }^{\mathbf{1}}$

DOI: 10.1590/0103-1104202012514

RESUMO A dengue é um grave problema de saúde pública, principalmente em países que apresentam condi-
ções socioambientais propícias para o desenvolvimento e proliferação do seu agente transmissor. Este estudo
teve como objetivo analisar a distribuição espacial da incidência da dengue no estado da Paraíba entre 2007 e
2016, avaliando a existência de dependência geográfica e sua relação com fatores socioeconômicos e ambien-
tais. Estudo ecológico, tendo com unidade de análise os 223 municípios do estado. Utilizaram-se o Índice de
Moran global e local e a estatística c de Geary para avaliar a autocorrelação espacial da dengue e a associação
com variáveis socioambientais. As análises foram realizadas por meio do software estatístico R Core Team
versão 3.3.2. Ao analisar a distribuição de casos de dengue nos municípios da Paraíba, pode-se identificar que
a doença vem avançando e acometendo maior número de cidades a cada ano. A doença esteve presente em
todos as cidades analisadas, o que demonstrou a necessidade de implementar ações de prevenção da doença
em todo o estado. Mediante esta pesquisa, ficou claro que a dengue, no estado da Paraíba, não é determinada
por fator único e isolado, mas sim, pela combinação de vários fatores do contexto socioeconômico e ambiental.

PALAVRAS-CHAVE Dengue. Fatores socioeconômicos. Indicadores ambientais. Análise espacial. Epidemiologia.

\begin{abstract}
Dengue is a serious public health problem, especially in countries that have favorable socio-environmental conditions for the development and proliferation of its transmitting agent. This study aimed to analyze the spatial distribution of the incidence of dengue in the state of Paraiba between 2007 and 2016, evaluating the existence of geographic dependence and its relationship with socioeconomic and environmental factors. Ecological study, with the unit of analysis of the 223 municipalities in the state. The global and local Moran Index and the Geary c statistic were used to assess the spatial autocorrelation of dengue and the association with socioenvironmental variables. The analyzes were performed using statistical software $R$ Core Team version 3.3.2. When analyzing the distribution of dengue cases in the municipalities of Paraiba, it can be identified that the disease has been advancing and affecting a greater number of cities each year. The disease was present in all the cities analyzed, which demonstrated the need to implement disease prevention actions across the state. Through this research, it became clear that dengue, in the state of Paraíba, is not determined by a single and isolated factor, but, rather, by the combination of several factors of the socioeconomic and environmental context.
\end{abstract}

1 Universidade Estadual da Paraíba (UEPB) - João Pessoa (PB), Brasil. enf.ellentay@gmail.com

2 Universidade Federal de Lavras (Ufla) - Lavras (MG), Brasil.
KEYWORDS Dengue. Socioeconomic factors. Environmental indicators. Spatial analysis. Epidemiology. 


\section{Introdução}

A dengue é uma enfermidade infecciosa aguda sistêmica de etiologia viral, causada por quatro sorotipos do Vírus da Dengue (Denv) (Denv-1, Denv-2, Denv-3 E Denv-4) pertencentes ao gênero Flavivirus e à família Flaviviridae $\mathbf{1}^{\mathbf{1 , 2}}$. Sua transmissão se dá principalmente pela picada de mosquitos fêmeas do gênero Aedes, sendo o Aedes aegypti o vetor primário, o qual se encontra distribuído nas regiões tropicais e subtropicais do mundo, predominantemente em áreas urbanas e semiurbanas ${ }^{3}$.

Durante os últimos 50 anos, a incidência de dengue aumentou 30 vezes, com ampliação da expansão geográfica para novos países, pequenas cidades e áreas rurais ${ }^{4,5}$. Estimativas sugerem que, por ano, ocorrem 390 milhões de infecções causadas pelos vírus da dengue, dos quais 96 milhões apresentam manifestações clínicas $^{2}$. Nas regiões das Américas, a doença tem-se disseminado com surtos ocorrendo em períodos de 3-5 anos, com registros de 561,501 casos confirmados e 1,181 óbitos em $2015^{4}$. No Brasil, em 2016, ocorreram 1.496 .282 casos de dengue (725,24/100.000 habitantes), sendo 844 casos considerados graves, 8.237 casos com sinais de alarme e 629 óbitos confirmados ${ }^{6}$.

Trata-se, portanto, de um grave problema de saúde pública, principalmente em países que apresentam condições socioambientais propícias para o desenvolvimento e a proliferação do agente transmissor ${ }^{7}$. Incluemse, nesse espectro, fatores como ausência de educação ambiental, baixa vigilância epidemiológica, manejo inadequado de resíduos sólidos, abastecimento de água intermitente ou esgotamento sanitário precário. Esses fatores combinados com o crescimento populacional e urbano, aliado à desinformação dos moradores quanto à necessidade de eliminar os focos de proliferação, potencializam a ocorrência de dengue nos espaços urbanos 8-11.

A análise espacial se mostra como uma ferramenta da informática em saúde muito útil para o estudo das dinâmicas de difusão espacial e temporal que permite observar áreas com maior risco epidemiológico, tanto pela localização de focos com potencial de proliferação do vetor como pelos fatores sociais e demográficos, que inevitavelmente predizem a ocorrência de doença ${ }^{12}$. Possibilitam o conhecimento de áreas e períodos com maior risco, a fim de produzir alertas para as vigilâncias epidemiológica e ambiental, tanto para o controle do vetor quanto na organização da assistência necessária ao atendimento dos casos. Nesse sentido, modelos estatísticos que levam em consideração a dependência espacial podem relacionar a incidência da doença com fatores ambientais e socioeconômicos de risco ${ }^{13}$.

Chen et al. ${ }^{14}$ estudaram os impactos individuais e interativos dos fatores socioecológicos sobre a dengue na China. No México, ReyesCastro et al. ${ }^{15}$ estudaram a propagação de um surto de dengue com base no agrupamento áreas de alta densidade e clusters. Análises espaçotemporais de casos de dengue foram realizadas em Manzanillo (município de Itagüí) ${ }^{16}$, na Argentina ${ }^{17}$ e na Colômbia ${ }^{18}$. No Brasil, foram identificadas poucas publicações que discutem dengue e associação com variáveis climáticas e sociodemográficas. $\mathrm{Em}$ Salvador ${ }^{19}$, Bahia, estudo avaliou se as comunidades urbanas estão associadas ao risco de dengue. Ribeiro et al. ${ }^{\mathbf{2 0}}$ avaliaram as desigualdades socioeconômicas e a dengue no Distrito Federal. Honorato et al. ${ }^{21}$, no Espírito Santo, e Almeida et al. ${ }^{22}$, para a cidade do Rio de Janeiro, mostraram haver associação positiva entre maiores riscos de transmissão de dengue e piores níveis socioeconômicos da população.

Não foi encontrado nenhum estudo sobre a detecção de dependência geográfica no estado da Paraíba, por meio da utilização de modelos estatísticos. Nesse contexto, o estado é um cenário importante para a compreensão dos fatores que afetam a dinâmica da transmissão da dengue e a exacerbação da doença, uma vez que a Paraíba apresenta consideráveis diferenças socioeconômicas e demográficas entre suas regiões administrativas, além de apresentar altas taxas de incidência ao longo dos anos. 
A partir do exposto, este estudo se propõe a analisar a distribuição espacial dos coeficientes de incidência da dengue no estado da Paraíba, avaliando a existência de dependência geográfica e sua relação com fatores socioeconômicos e ambientais.

\section{Metodologia}

Trata-se de um estudo epidemiológico do tipo ecológico para traçar a distribuição espacial dos casos notificados por dengue no estado da Paraíba entre 2007 e 2016, levando-se em consideração todos os seus 223 municípios. Foram utilizados os dados do Sistema de Informação de Agravos de Notificação (Sinan), disponibilizados pela Gerência Executiva de Vigilância em Saúde da Secretaria de Estado da Saúde da Paraíba. As informações sobre condições socioeconômicas são provenientes do Censo Demográfico de 2010, realizado pelo Instituto Brasileiro de Geografia e Estatística ${ }^{23}$; e os relativos a variáveis ambientais foram cedidos pela Climate (www.climate-data.org).

O estado da Paraíba, localizado na região Nordeste, apresenta uma área de $56.439 \mathrm{~km}^{2}$, que corresponde a $0,662 \%$ do território brasileiro. Apresentava uma população estimada de 3.996.496 milhões de habitantes em 2018 e encontra-se entre os paralelos $6^{\circ} 02$ ' 12 " e $8^{\circ} 19^{\prime} 18$ "'S, e entre os meridianos de $34^{\circ} 45^{\prime} 54^{\prime \prime}$ e $38^{\circ} 45^{\prime} 45^{\prime \prime} \mathrm{W}^{13}$. Devido a sua proximidade com a linha do Equador, possui um clima quente, com temperaturas elevadas, variando em função do relevo. O estado é constituído por quatro mesorregiões, a saber: Zona da Mata; Agreste paraibano; Borborema; e Sertão paraibano ${ }^{\mathbf{2 4}}$.

Utilizou-se como variável dependente o Coeficiente Médio de Incidência por dengue, calculado a partir do número total de casos de cada arbovirose notificado em diferentes períodos, divididos pelo respectivo total de pessoas-ano e multiplicados por 100 mil. Para a caracterização do contexto socioeconômico e ambiental, utilizaram-se como variáveis independentes as seguintes: Índice de Desenvolvimento Humano;
Índice de Gini; precipitação média anual; temperatura média anual em graus Celsius; proporção de domicílios com mais de oito residentes; proporção de domicílios sem renda mensal; proporção de domicílios com eletricidade de origem desconhecida; proporção de domicílios em que o lixo é jogado em terreno baldio ou logradouro público; proporção de municípios com coleta de lixo; proporção de domicílios ligados à rede geral de esgoto ou com fossa séptica; proporção de domicílios com saneamento básico (abastecimento de água adequado, esgotamento sanitário adequado e destino do lixo adequado); proporção de domicílios particulares ligados à rede geral de água; proporção de indivíduos alfabetizados; e taxa de desemprego.

Para analisar a autocorrelação espacial, utilizaram-se duas medidas que são amplamente recomendadas para dados de área e variáveis em escala intervalar. A primeira, o Índice I de Moran, é uma medida que fornece a significância estatística da autocorrelação espacial entre as áreas dentro da região de estudo. Esse índice varia de -1 a 1, quanto mais próximo dos extremos indica autocorrelação significativa, quanto mais próximo de zero indica completa aleatoriedade. A segunda medida, a estatística c de Geary, possibilita a avaliação da existência de associação entre os valores de um determinado atributo entre as áreas de uma região de estudo, levando-se em consideração a localização espacial dessas áreas. Esse parâmetro varia em um intervalo de 0 a 2, em que 0 significa associação perfeita, 1 representa um comportamento aleatório e 2 indica dispersão perfeita.

Ainda que essas medidas globais sejam muito úteis para fornecer uma indicação de agrupamento global dos dados, foram complementadas por estatísticas locais, com objetivo de avaliar a presença de clusters, por meio do Índice de Moran Local (Local Indicators of Spatial Association - Lisa). Além disso, para analisar a variação de tendência espacial da taxa de pessoas infectadas pelo Aedes aegypti, foi utilizada a Média Móvel Local para cada município em estudo. 
Para verificar a similaridade dos valores do atributo das áreas na região de estudo de maneira visual, foram construídos o gráfico de espalhamento de Moran, os mapas de Box Map, Lisa Map, Moran Map e o mapa do índice de Moran local. Essas figuras fornecem informações acerca da associação entre os valores do atributo nas áreas com seus vizinhos.

O gráfico de espalhamento de Moran ou diagrama de espalhamento de Moran é uma maneira adicional de visualizar a dependência espacial. Construído com base nos valores normalizados (valores de atributos subtraídos de sua média e divididos pelo desvio padrão), permite analisar o comportamento da variabilidade espacial. É calculado pela autocorrelação espacial entre os vizinhos. A análise dos quadrantes do diagrama indica áreas de associação espacial positiva Q1 (valores positivos, médias positivas) e Q2 (valores negativos, médias negativas), e áreas de associação espacial negativa Q3 (valores positivos, médias negativas) e Q4 (valores negativos, médias positivas). As áreas localizadas nos quadrantes Q1 e Q2 apresentam autocorrelação positiva, ou seja, as áreas formam clusters de valores similares. As áreas localizadas nos quadrantes Q3 e Q4 apresentam autocorrelação negativa, isto é, há dissimilaridade entre as áreas vizinhas.

O Box Map apresenta os clusters, independentemente da signifi-cância estatística; o Lisa Map indica regiões com correlação local significativamente diferente das demais; enquanto o Moran Map, apenas aqueles com valor de $\mathrm{p}<0,10$. O mapa do Índice de Moran local fornece as áreas que têm valores similares e dissimilares aos seus vizinhos, em que valores positivos do índice indicam áreas com valores similares para o atributo, e valores negativos do índice indicam dissimilaridade entre os valores dessas áreas.

Com o intuito de verificar o melhor modelo explicativo de associação das variáveis do contexto socioeconômico e ambiental, foram aplicadas quatro alternativas de modelos de regressão: regressão clássica; espacial autorregressivo; erro espacial; e o modelo espacial de Durbin. O Modelo de Regressão Clássica (MRC) baseia-se no relacionamento entre duas ou mais variáveis de forma que uma delas possa ser explicada ou ter seu valor predito por meio de outras variáveis ${ }^{25}$. No caso de dados espaciais, havendo autocorrelação espacial, o modelo gerado deve incorporar a estrutura espacial, pois a dependência entre as observações afeta a capacidade de explicação do modelo ${ }^{26}$.

A suposição de que as observações sejam independentes simplifica o modelo, mas, no contexto de dados de área, essa simplificação é pouco provável que aconteça devido à possibilidade de dependência espacial entre os termos do erro. Uma alternativa, nessas circunstâncias, é utilizar o Modelo Espacial Autorregressivo (Mear) que atribui à variável resposta $Y$ a autocorrelação espacial ignorada. O Mear é dado pela seguinte equação: $Y=X \beta+$ $\rho W Y+\varepsilon$, em que: $W$ é a matriz de proximidade espacial; $W Y$ expressa a dependência espacial em $Y$; e $\rho$ é o coeficiente espacial autorregressivo ${ }^{27}$. Nesse modelo, a autocorrelação espacial é incorporada como componente do modelo.

Outra alternativa é o ajuste do Modelo do Erro Espacial (MEE), que considera os efeitos espaciais como um ruído, isto é, como um fator a ser removido. Esse modelo parte do princípio de que não é possível modelar todas as características de uma unidade geográfica que podem influenciar as regiões vizinhas. Os efeitos da autocorrelação espacial estão associados ao termo de erro $\varepsilon$ e o modelo é descrito por: $Y=X \beta+\varepsilon, \varepsilon=\lambda W \varepsilon+\xi$, em que: $W \varepsilon$ é a componente do erro com efeitos espaciais; $\lambda$ é o coeficiente autoregressivo; $\xi$ é a componente do erro com variância constante e não correlacionada ${ }^{28}$.

O Modelo Espacial de Durbin (MED) é uma extensão desses modelos anteriores na qual é incluído o 'spatial lag' na variável dependente $Y$ e, também, na variável independente: $Y=\rho W Y+X \beta+W X \theta+\varepsilon$, em que: $Y$ contém um vetor de variáveis dependentes e $X$ representa a matriz de dados contendo as variáveis independentes com um vetor de parâmetro associado $\beta$; $W$ é a matriz de peso espacial e o parâmetro $\rho$ é um coeficiente de 'spatial lag' 
da variável resposta. Um conjunto adicional de variáveis explicativas é adicionado ao modelo construindo um intervalo espacial das variáveis explicativas usando o produto da matriz $W X$, com os parâmetros associados $\theta$. Esse conjunto representa variáveis explicativas construídas como médias de observações vizinhas.

O teste de correlação linear de Spearman foi aplicado para verificar as correlações entre as variáveis independentes e o Coeficiente Médio de Incidência por dengue $(p<0,001)$. $\mathrm{Na}$ avaliação de qual modelo melhor se ajustaria às variáveis disponíveis, foi escolhido aquele que apresentou menor valor da log-verossimilhança e menores valores do critério de informação de Akaike (AIC).

As análises foram realizadas por meio do software estatístico R Core Team versão 3.3.2 de 2017(http://www.rproject.org/), com auxílio dos pacotes geoR, POT, spatial extremes, spatial e spdep.

\section{Resultados}

No período de janeiro de 2007 a dezembro de 2016, foram registrados 162.521 casos de dengue em residentes no estado da Paraíba. Na análise da distribuição espacial das incidências por município, as maiores foram aquelas registradas no Sertão do estado, nos municípios de Princesa Izabel (2.136 casos/100 mil hab.), Carrapateira (2.056 casos/100 mil hab.) e Monte Horebe (2.036 casos/100 mil hab.), bem como no município de Monteiro (2.053 casos/100 mil hab.), localizado no Agreste paraibano, com pequena densidade populacional (figura 1).

Figura 1. Coeficiente de incidência por dengue nos 223 municípios do estado da Paraíba por 100 mil habitantes, 20072016

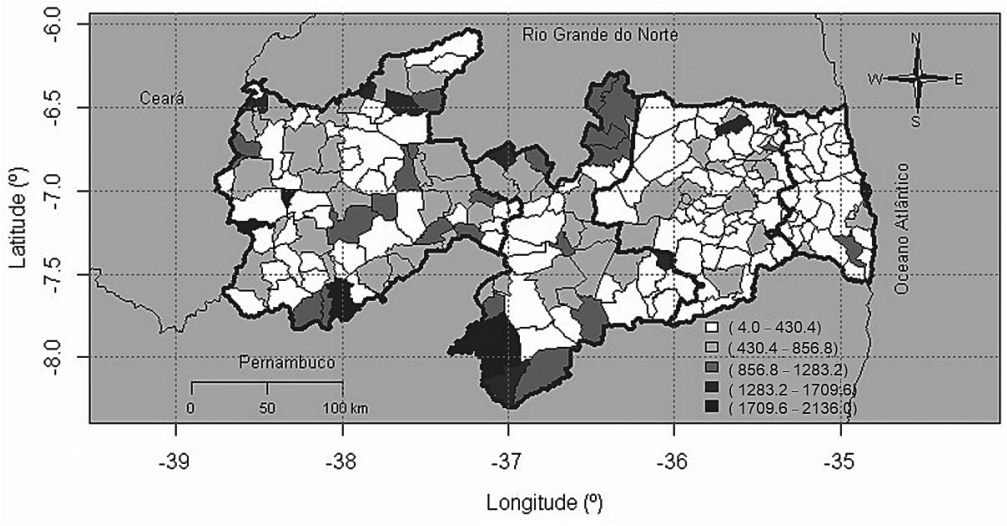

Fonte: Secretaria Estadual de Saúde da Paraíba/MS.

Para reconhecer o padrão da distribuição espacial dos casos notificados por dengue, foi inicialmente estimada a magnitude da autocorrelação espacial entre as áreas, pelo Índice de Moran Global, sob a hipótese nula de ausência de autocorrelação. Na análise da variável dependente, o Índice de Moran Global encontrado foi de 0,20510 ( $p<0,001)$, identificando uma dependência no Coeficiente Médio de Incidência por dengue entre municípios vizinhos. A partir do teste de aleatoriedade espacial de Geary, obteve-se a estatística calculada igual a 0,73434 que corresponde a um valor $\mathrm{p}<0,001$, identificando autocorrelação espacial significativa.

A figura $2 A$ mostra um mapa da Média 
Móvel Local construído para analisar a tendência espacial da taxa de pessoas notificadas por dengue. Verifica-se que, à medida que se aproxima do interior do estado, há um aumento na média móvel, indicando existir uma tendência espacial mais fortemente observada nessa localidade. Por meio do Índice de Moran Local (figura $2 B$ ), pode-se verificar o quanto as áreas vizinhas são similares, identificando que grande parte dos municípios da Mata paraibana têm valores similares de casos notificados por dengue.

Figura 2. Média Móvel Local (2A) e Índice de Moran Local (2B) do Coeficiente Médio de Incidência por dengue no estado da Paraíba, 2007-2016

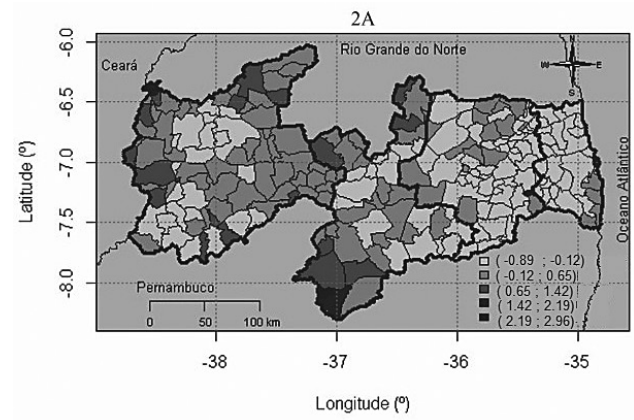

Fonte: Secretaria Estadual de Saúde da Paraíba/MS.

Por meio do Lisa Map (figura $3 A$ ) e do Moran Map (figura 3B), é possível observar que os municípios que apresentaram maior significância estatística para a autocorrelação espacial foram Monteiro, São Sebastião do Umbuzeiro, Joca Claudino, Princesa Isabel e Zabelê. Ainda, no Moran Map, é possível

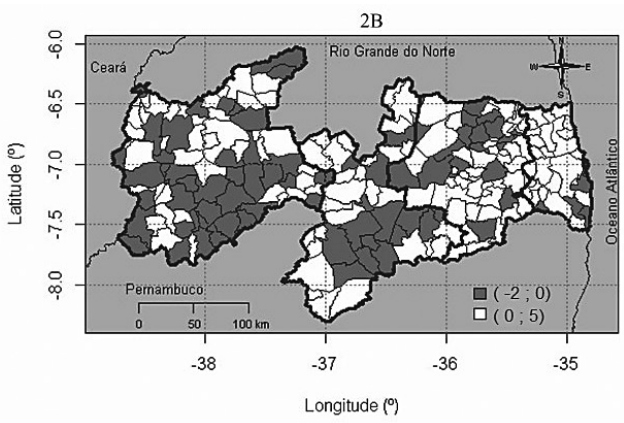

enxergar os municípios que tiveram autocorrelação espacial significativa ao nível de 10\%: Picuí; Bom Jesus; Brejo dos Santos; Massaranduba; Riachão do Bacamarte; Caiçara; Caldas Brandão; e alguns municípios do Litoral Norte paraibano, como Mataraca, Baía da Traição, Rio Tinto e Lucena.

Figura 3. Lisa Map (3A) e Moran Map (3B) do Coeficiente Médio de Incidência por dengue no estado da Paraíba, 20072016
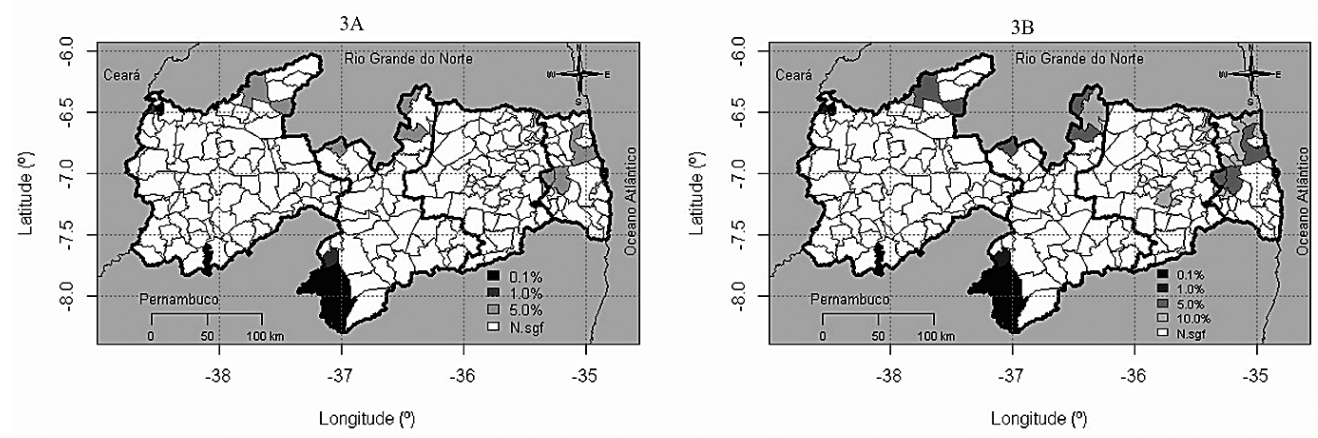
Observaram-se, no gráfico de espalhamento de Moran (figura 4A), primeiro e segundo quadrantes, municípios com associação espacial positiva, no sentido que uma localização possui vizinhos com valores semelhantes. Os municípios Bernardinho Batista, Brejo dos Santos, São Sebastião do Umbuzeiro, Zabelê, Monteiro, Riacho dos Cavalos e Monte Horebe são preocupantes, pois, além de apresentarem altos coeficientes de incidência por dengue, estão cercados por outros municípios que também apresentam altos coeficientes. $\mathrm{Na}$ extensão do espalhamento de Moran, no Box Map (figura 4B), municípios representados por cores mais escuras (por exemplo, Zabelê,
Monteiro, São Sebastião do Umbuzeiro, Brejo dos Santos e Riacho dos Cavalos) apresentaram autocorrelação espacial positiva (figura $4 A$ ). Por outro lado, os municípios representados por tonalidades mais claras (por exemplo, Dona Inês, Carrapateira e Princesa Isabel) apresentaram dissimilaridade com seus vizinhos em relação aos coeficientes de incidência de dengue no estado da Paraíba. Sendo assim, observa-se que a maioria dos municípios têm valores similares aos seus vizinhos para o coeficiente de incidência de pessoas notificadas por dengue, expondo, assim, a importância de levar em consideração dependência espacial deles.

Figura 4. Mapa de espalhamento de Moran (4A) e Box Map (4B) da análise de autocorrelação espacial dos coeficientes de incidência por dengue no estado da Paraíba, 2007-2016

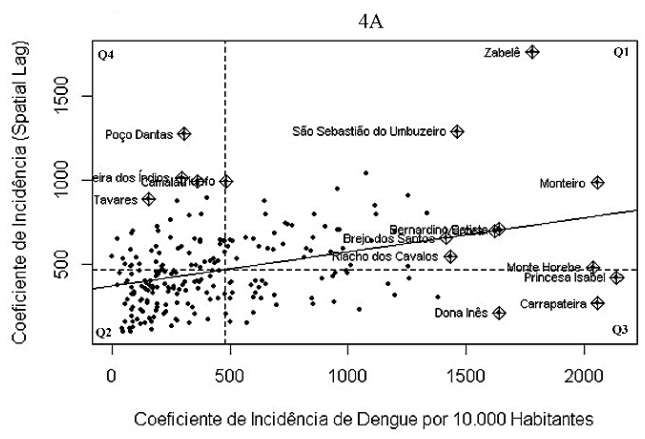

Fonte: Secretaria Estadual de Saúde da Paraíba/MS.

Nas análises de associação das variáveis do contexto socioeconômico e ambiental (dados não apresentados em tabela), foi possível verificar que a proporção de domicílios com saneamento básico $(-0,3022 ; \mathrm{p}<0,05)$, proporção de municípios com coleta de lixo $(-0,6445$; $p$ $<0,001)$ e proporção de municípios com rede geral de esgoto ou com fossa séptica $(-0,6128 ; \mathrm{p}<$ $0,001)$ apresentaram correlação estatisticamente significante e inversamente proporcional com o Coeficiente Médio de Incidência por dengue. Mostraram correlação significativa positiva as seguintes variáveis: Índice de Desenvolvimento

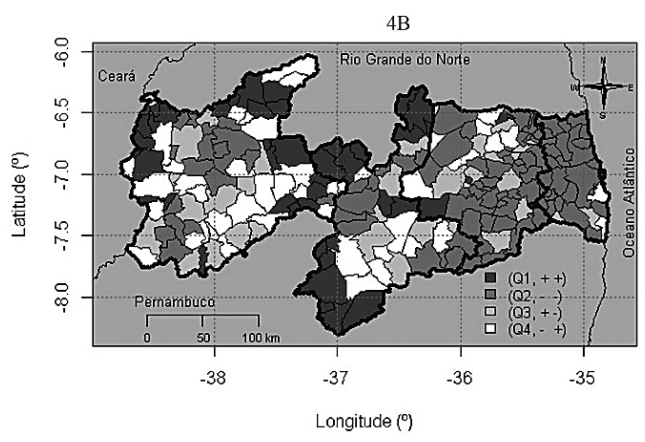

Humano (0,4114; $\mathrm{p}<0,001)$; Índice de Gini (0,4498; $\mathrm{p}<0,001)$; proporção de domicílios em que o lixo é jogado em terreno baldio ou logradouro público $(0,3840 ; \mathrm{p}<0,001)$.

Analisando-se os resultados apresentados na tabela 1, pode-se observar que o modelo que melhor se ajustou aos dados foi o Mear, pois ele apresentou o menor valor de AIC. Segundo o modelo, o Índice de Desenvolvimento Humano do município apresentou contribuição significativa para a incidência de dengue no estado, enquanto o Índice de Gini e a proporção de indivíduos alfabetizados apresentaram correlação inversa. 
Tabela 1. Ajustes dos modelos de regressão para o Coeficiente Médio de Incidência por dengue no estado da Paraíba, 2007-2016, com variáveis socioeconômicas e ambientais

\begin{tabular}{lrrrr}
\hline Covariáveis & RC $(p-$ valor $)$ & Mear $(p-$ valor $)$ & MEE $(p-$ valor $)$ & MED $(p$ - valor $)$ \\
\hline Intercepto & $-1450,0(<0,01)$ & $-1417,6(<0,01)$ & $-1301,1(0,0111)$ & $-2464,7(0,0172)$ \\
IDH & $5209,0(<0,01)$ & $4599,8(<0,01)$ & $4777,5(<0,01)$ & $4266,1(<0,01)$ \\
Gini & $-2565,0(0,0128)$ & $-2427,5(0,0127)$ & $-2227,8(0,0227)$ & $-1696,1(0,0952)$ \\
Precip & $-0,1801(0,0641)$ & - & $-0,2013(0,0799)$ & $-0,4264(0,0634)$ \\
PA & $-0,0015(0,0694)$ & $-0,0016(0,0429)$ & $-1272,9(0,0966)$ & $-0,0015(0,0539)$ \\
Temp $\left({ }^{\circ} \mathrm{C}\right)$ & - & - & $-0,0104(0,0085)$ \\
AIC & 3307,976 & $-3302,127$ & 3303,643 & 3302,508 \\
\hline$\rho$ & $0,87(<0,001)$ & $11,662(<0,153)$ & $7,300(0,072)$ & $6,332(0,092)$ \\
\hline Resíduo & & &
\end{tabular}

Fonte: Secretaria Estadual de Saúde da Paraíba/MS.

RC: Regressão Clássica; Mear: Modelo Espacial Autorregressivo; MEE: Modelo de Erro Espacial; MED: Modelo Espacial de Durbin; IDH: Índice de Desenvolvimento Humano; Precip: Precipitação Média Anual; PA: População Alfabetizada; Temp: Temperatura Média Anual.

\section{Discussão}

Os municípios que compõem a região do Sertão paraibano destacaram-se neste estudo pelas altas taxas de incidência. Quanto aos possíveis fatores explicativos para a distribuição de dengue, a seca é considerada um fator fundamental, em que ambientes com períodos de estiagens e secas podem causar um abastecimento irregular de água, o que pode levar ao armazenamento inadequado e, por consequência, propiciar criadouros ao vetor ${ }^{29}$.

A densidade populacional em estudos realizados no Espírito Santo ${ }^{21}$, no Rio de Janeiro ${ }^{22}$ e em duas cidades do México ${ }^{15}$ contribuiu positivamente para a taxa de incidência da dengue. Eles destacam que ambientes mais populosos favorecem a proliferação do vetor, apresentando condições ideais para sua reprodução, bem como para o maior número de indivíduos suscetíveis a novas infecções ${ }^{30}$, em discordância com os resultados deste estudo, no qual os municípios com pequena densidade populacional foram os que apresentaram as maiores incidência do estado, o que corrobora Barbosa e Silva ${ }^{31}$ e Machado et al. ${ }^{32}$ que identificaram uma baixa correlação espacial entre densidade populacional e a taxa de incidência de dengue. Segundo Almeida e Medronho ${ }^{22}$, não necessariamente todas as áreas de alta densidade populacional estão associadas a taxas de incidência de dengue, podendo outros fatores estarem relacionados, como os aspectos sociodemográficos e ambientais, que poderiam explicar melhor essas incidências.

É possível identificar vários indicadores de condições socioeconômicas que podem interferir na saúde de uma população. Neste estudo, o Índice de Desenvolvimento Humano apresentou correlação espacial positiva, permitindo observar áreas com melhores valores de Índice de Desenvolvimento Humano Municipal (IDHM) e altos coeficientes de incidência por dengue, em concordância com estudo realizado no estado do Maranhão ${ }^{33}$, que destacou que os municípios com os maiores valores de IDHM são provavelmente os mais populosos, com maior nível de desenvolvimento, melhor infraestrutura sanitária e maior acesso aos serviços de saúde, todos contribuindo para uma maior notificação dos casos de dengue.

O índice de Gini apresentou correlação espacial negativa com as taxas de incidência da dengue, permitindo observar um aumento na incidência em áreas com melhores valores 
do índice. Esse achado discorda da pesquisa de Ribeiro et al. ${ }^{20}$ que avaliou as desigualdades socioeconômicas e a dengue no Distrito Federal. No entanto, a relação do Gini com o Aedes Aegypti ainda causa discordância nos estudos. Paula et al. ${ }^{\mathbf{3 4}}$ e Ribeiro et al. ${ }^{20}$ encontraram as maiores taxas em áreas carentes. Por outro lado, Resende et al. ${ }^{35}$ e Almeida et al.22 encontraram alta incidência tanto em áreas com precárias condições quanto nas mais desenvolvidas. Outras pesquisas realizadas com dengue não identificaram relação do vetor com os índices de Gini. Essa discordância pode estar relacionada com a heterogeneidade da transmissão dessa doença.

O destino inadequado do lixo, a proporção de municípios com rede geral de esgoto, a proporção de domicílios particulares ligados à rede geral de água e a taxa de desemprego também podem explicar a incidência da dengue no estado da Paraíba. Estudos anteriores, realizados em diversas regiões do mundo, também evidenciaram associações entre risco de ocorrência de dengue e níveis socioeconômicos. Honorato et al.21 no Espírito Santo, Chen et al. ${ }^{14}$ em Cantão na China, Almeida et al. ${ }^{22}$ para a cidade do Rio de Janeiro, e IraldoHurtado et al. ${ }^{16}$ em Manzanillo, município de Itagüí, mostraram haver associação positiva entre maiores riscos de transmissão de dengue e piores níveis socioeconômicos da população.

Por outro lado, em estudos realizados por Gil et al. ${ }^{17}$ em San Ramón de la Nueva Orán-AR, por Costa et al. ${ }^{36}$ em Campinas-SP e por ReyesCastro et al. ${ }^{15}$ no México, não foi encontrada correlação entre o percentual da população com necessidades básicas insatisfeitas e incidência de dengue, sendo considerado por eles que a falta de correlação entre casos e variáveis socioeconômicas é principalmente devido à pequena dimensão de alguma cidade, à distribuição generalizada da pobreza e à alta mobilidade dos habitantes dentro da cidade, o que poderia tornar o risco homogêneo.

Neste estudo, não foi identificada correlação significativa entre a incidência da dengue com a pluviosidade e a temperatura, enquanto no estado de Goiás, a situação estabelecida está diretamente relacionada com os fatores abióticos que favorecem a existência de criadouros disponíveis para o desenvolvimento do vetor ${ }^{37}$. Estudo realizado em Boa Vista - Roraima, também não demonstrou correlação entre as variáveis meteorológicas ${ }^{38}$, diferentemente do cenário mais amplo do País, onde a maioria dos casos ocorre no período chuvoso.

Os resultados discordantes entre as associações encontradas em diferentes pesquisas podem estar relacionados com o tipo de unidade espacial utilizada (setor censitário, bairros, distritos e/ou municípios). Outra possibilidade seria uma limitação resultante da agregação espacial, já que, dependendo do nível de agregação, a captação da realidade pode não ser a ideal, ou seja, dependendo do modo como as variáveis foram agregadas para descrever extensas regiões, podem produzir grande variação de resultados ${ }^{39}$.

A fim de levar em consideração a dependência espacial das variáveis de interesse, foram utilizados modelos ajustados aos dados, sendo capazes de identificar a influência de algumas variáveis independentes. Outras variáveis importantes para a transmissão da doença - como, por exemplo, a regularidade do abastecimento de água, a pluviosidade e a temperatura - poderiam ter sido levadas em consideração.

Com relação aos resíduos dos modelos, para o MRC, pode-se observar que ele não seguiu um comportamento normal ( $\mathrm{p}$-valor $<0,05$ ), uma vez que esse modelo não prevê o ajuste da dependência espacial entre os municípios estudados. No entanto, ao se utilizar os modelos de regressão espacial, observou-se independência na estrutura da autocorrelação residual espacial ( $\mathrm{p}$-valor $>0,05)$. Em geral, modelos de regressão espacial, que levam em consideração a variação dos parâmetros no espaço, são mais eficientes do que os modelos de regressão com efeitos globais. Os modelos de efeitos globais partem do pressuposto que o processo espacial é estacionário, isto é, a autocorrelação espacial é estimada por meio de um único parâmetro. 
Entretanto, a utilização de dados censitários pode produzir padrões espaciais diversos que não são captados em um único parâmetro ${ }^{40}$.

$\mathrm{O}$ estudo apresenta limitações inerentes à utilização de dados secundários, uma vez que as informações foram baseadas em registros de casos notificados do Sinan, sendo assim, registrados apenas os casos de evolução grave, que buscam uma unidade de saúde para atendimento, podendo haver subnotificação das formas leves da doença. Mesmo diante dessa limitação, os achados do presente estudo são fundamentais para elencar possíveis fatores de risco, associados à ocorrência da dengue no estado da Paraíba, assim como identificar municípios prioritários para o desenvolvimento de ações de controle da doença.

Ao analisar a distribuição de casos de dengue nos municípios do estado da Paraíba, pode-se identificar que a doença vem avançando e acometendo maior número de cidades a cada ano. A doença esteve presente em todos as cidades analisadas, o que demonstrou a necessidade de implementar ações de prevenção da dengue em todo o estado.

\section{Conclusões}

Diante desta pesquisa, ficou claro que a dengue, no estado da Paraíba, não é determinada por fator único e isolado, mas, sim, pela combinação de vários fatores do contexto socioeconômico e ambiental, como saneamento básico, Índice de Desenvolvimento Humano e Índice de Gini. O comportamento da população com hábitos inadequados de descarte de lixo e um possível armazenamento de água, diante do período de estiagem no Nordeste, também contribuem para a manutenção da doença.

Além disso, neste estudo, foi possível demonstrar que a análise da distribuição espacial é um importante instrumento a ser utilizado na monitorização dos casos de dengue e pode ser adotado pelos setores de vigilância epidemiológica e de controle do vetor dos municípios, auxiliando assim no planejamento de ações em áreas estratégicas de maior vulnerabilidade, gerando impacto positivo no controle do agravo e na diminuição da severidade da doença.

\section{Colaboradores}

Silva ETC (0000-0002-2079-6240)*, Olinda RA (0000-0002-0509-8428)*, Pachá AS (0000-0002-7020-5445)*, Costa AO (0000-0002-9212-0493)*, Brito AL (00000002-3439-500X)*, Pedraza DF (0000-00025394-828X) contribuíram igualmente para a elaboração do manuscrito. 


\section{Referências}

1. Dussart P, Lavergne A, Lagathu G, et al. Reemergence of dengue virus type 4, French Antilles and French Guiana, 2004-2005. Emerging infectious diseases. Emerg Infect Dis. 2006; 12(11):1748-1751.

2. Bhatt $\mathrm{S}$, Gething PW, Brady OL, et al. The global distribution and burden of dengue. Nature. 2013; 496(7446):504-507.

3. Halstead SB. Epidemiology of dengue and dengue hemorrhagic fever. In: Gubler DJ, Kuno G, organizador. Dengue and dengue hemorrhagic fever. New York: CAB International. 1998; 11(3):480-496.

4. Brasil. Ministério da Saúde, Secretaria de Vigilância em Saúde, Departamento de Vigilância das Doenças Transmissíveis. Febre de Chikungunya: manejo clínico. Brasília, DF; 2015.

5. World Health Organization. Global Strategy for Dengue Prevention and Control 2012-2020. Genebra: WHO; 2015.

6. Brasil. Ministério da Saúde, Secretaria de Vigilância em Saúde. Monitoramento dos casos de dengue, febre de chikungunya e febre pelo vírus Zika até a Semana Epidemiológica 52. Boletim Epidemiológico. 2018 [acesso em 2020 maio 18]; 50(4). Disponível em: http://www.saude.gov.br/images/pdf/2017/ abril/06/2017-002-Monitoramento-dos-casos-de-dengue--febre-de-chikungunya-e-febre-pelo-v--rus-Zika-ate-a-Semana-Epidemiologica-52--2016.pdf.

7. Lopes N, Linhares REC, Nozawa C. Características gerais e epidemiologia dos arbovírus emergentes no Brasil. Rev Pan-Ama Saude. 2014; 5(3):55-64.

8. Gubler DJ. Dengue and dengue hemorrhagic fever: its history and resurgence as a global health problem. In: Gubler DJ, Kuno G, orgnizadores. Dengue and dengue hemorrhagic fever. New York: CAB International; 1997. p. 1-22.

9. Silva AA, Miranda CF, Ferreira JR, et al. Fatores sociais e ambientais que podem ter contribuído para a proliferação da dengue em Umuarama, estado do Paraná. Acta Scientiarum Health Sciences. 2003; 25(1):81-85.

10. Barbosa IR, Silva LP. Influência dos determinantes sociais e ambientais na distribuição espacial da dengue no município de Natal-RN. Rev. Ciênc. Plur. 2015; $1(3): 62-75$.

11. Pelissari B. Aspectos socioambientais associados à ocorrência de dengue em um município do estado do Mato Grosso. Rev. epidemiol. controle infecç. 2016; 6(1):12-17.

12. Skalinski LM, Costa MCN, Lima TMG. Contribuições da análise espacial para a compreensão da dinâmica de transmissão da dengue: revisão integrativa. JHBS. 2019; 7(53):53-63.

13. Olinda RA, Ozaki VA, Juliette B, et al. Estudo de simulação de extremos espaciais com base em processos max-stable. RBB. 2013; 31(1):132-156.

14. Chen B, Yang J, Luo L, et al. Who is vulnerable to dengue fever? A community survey of the 2014 outbreak in Guangzhou, China. Int J Environ Res Public Health. 2016; 13(7):712.

15. Reyes-Castro PA, Harris RB, Brown HE, et al. Spatio-temporal and neighborhood characteristics of two dengue outbreaks in two arid cities of Mexico. Acta Trop. 2017; 167:174-182.

16. Iraldo-Hurtado TM, Alvarez-Betancur JP, Parra-Henao G. Factores asociados a la infestación domiciliaria por Aedes aegypti en el corregimiento el Manzanillo, municipio de Itagüí (Antioquia) año 2015. Rev. Fac. Nac. Salud Pública. 2018; 36(1):34-44.

17. Gil JF, Palacios M, Krolewiecki AJ, et al. Spatial spread of dengue in a non-endemic tropical city in northern Argentina. Acta tropica. 2016; 158:24-31.

18. González AAM, Ortiz Beltrán FG, Santa Guzmán LF. Modelo bayesiano para el estudio de la enfermedad 
del dengue en el departamento de Atlántico, Colombia, años 2010 a 2013. Perspect Geográfica [internet]. 2017 [acesso em 2020 abril 27]; 22(2). Disponível em: http://revistas.uptc.edu.co/revistas/index.php/perspectiva/article/view/7603.

19. Kikuti M, Cunha GM, Paploski IAD, et al. Spatial distribution of dengue in a Brazilian Urban slum setting: Role of socioeconomic gradient in disease risk. PLoS Negl Trop Dis. 2015; 9(7):1-18.

20. Ribeiro NG, Araújo HF, Lacerda LF, et al. Relação entre as condições socioeconômicas e as taxas de incidência de dengue nas regiões administrativas do Distrito Federal. Rev Med Saude Brasília. 2016; 5(1):22-27.

21. Honorato T, Lapa PP, Sales CM, et al. Análise espacial do risco de dengue no Espírito Santo, Brasil, 2010: uso de modelagem completamente Bayesiana. Rev Bras Epidemiol. 2014; 17(supl2):150-159.

22. Almeida AS, Medronho RA, Valencia LI. Spatial analysis of dengue and the socioeconomic context of the city of Rio de Janeiro (Southeastern Brazil). Rev Saude Publica. 2009; 43(4):666-673.

23. Instituto Brasileiro de Geografia e Estatística. Estimativas do Censo Demográfico: Indicadores Sociais Municipais. Rio de Janeiro: IBGE; 2018

24. Melo AST, Rodrigues JL. Paraíba: Desenvolvimento econômico e a questão ambiental, João Pessoa: Grafset; 2003.

25. Azevedo JVV, Santos CAC, Alves TLB, et al. Influence of climate on the incidence of acute respiratory infections in children at the districts of Campina Grande and Monteiro, Paraíba, Brazil. Rev. bras. Meteorol. 2015; 30(4):467-477.

26. Câmara G, Monteiro AMV, Medeiros JS. Fundamentos epistemológicos da ciência da geoinformação. In: Câmara G, Davis JR CA, Monteiro AMV, organizadores. Introdução à ciência da geoinformação. São José dos Campos: INPE; 2014. [acesso em 2018 ago 19]. Disponível em: http://www.dpi.inpe.br/gilberto/livro/introd/ cap5-epistemologia.pdf.
27. Chen Y. New approaches for calculating morans index of spatial autocorrelation. PloS one. 2013 [acesso em 2020 maio 18]; 8(7):e68336. Disponível em: https://journals.plos.org/plosone/article?id=10.1371/ journal.pone.0068336.

28. Fahl GB, Adamczyk WB. Incidência de dengue no Rio Grande do Sul: uma análise econométrica espacial; 2016.

29. Medeiros AMT, Brito AC. A seca no Estado da Paraíba - impactos e ações de resiliência. Parcerias Estratégicas, Brasília, DF. 2017; 22(44):139-154.

30. Scandar SA, Vieira P, Cardoso Junior RP, et al. Dengue em São José do Rio Preto, Estado de São Paulo, Brasil, 1990 a 2005: fatores entomológicos, ambientais e socioeconômicos. Bol Epidemiol Paulista. 2010; $7(81): 4-16$

31. Barbosa IR, Silva LP. Influência dos determinantes sociais e ambientais na distribuição espacial da dengue no município de Natal-RN. Rev Cienc Plural. 2015; 1(3):62-75.

32. Machado JP, Oliveira RM, Souza-Santos R. Análise espacial da ocorrência de dengue e condições de vida na cidade de Nova Iguaçu, Estado do Rio de Janeiro, Brasil. Cad. Saúde Pública. 2009; 25(5):1025-1034.

33. Costa SSB, Carvalho BMRF, Aquino Junior J, et al. Spatial analysis of probable cases of dengue fever, chikungunya fever and zika virus infections in Maranhão State, Brazil. Rev. Inst. Med. trop. S. Paulo. 2018 [acesso em 2020 maio 18]; 60:e62. Disponível em: https://www.scielo.br/scielo.php?script=sci_ abstract\&pid=S0036-46652018005000236\&lng=pt $\& \mathrm{nrm}=1 \&$ tlng=en.

34. Paula AR, Lima GA, Araújo BR, et al. A incidência de dengue associada a fatores socioeconômicos no município de campos dos Goytacazes-RJ. Revista Univap. 2016; 22(40):55-62.

35. Resende APC, Silveira NAPR, Sabroza PC, et al. Determinação de áreas prioritárias para ações de controle da dengue. Rev Saúde Pública. 2010; 44(2):274-282. 
36. Costa JV, Silveira LVA, Domalisio MR. Análise espacial de dados de contagem com excesso de zeros aplicado ao estudo da incidência de dengue em Campinas, São Paulo, Brasil. Cad. Saúde Pública. 2016 [acesso em 2020 maio 18]; 32(8):e00036915. Disponível em: https://www.scielo.br/scielo.php?pid=S0102$-311 X 2016000804003 \&$ script=sci_abstract\&tlng=pt.

37. Araújo RR, Nunes JS. Relações geográficas entre o clima e a incidência de dengue na cidade de São Luís-MA. Brasil. Ciências Humanas em Revista. 2005; 3(2):93-108.

38. Souza SS, Silva IG, Silva HHG. Associação entre incidência de dengue, pluviosidade e densidade larvária de Aedes aegypti, no Estado de Goiás. Revista da
Sociedade Brasileira de Medicina Tropical Rev. Soc.

Bras. Med. Trop. 2010; 43(2):152-155.

39. Silva RM, Silva AM, Chaves JJS. Vulnerabilidade espacial da dengue e sua relação com a variabilidade termo pluviométrica em João Pessoa-PB. Hygeia. 2014; 10(18):177-179.

40. Câmara G, Carvalho MS, Cruz OG, Correia V. Análise espacial de áreas. In: Druck S, Carvalho MS, Câmara G, Monteiro AMV, editores. Análise espacial de dados geográficos. Planaltina: Embrapa; 2004.

\footnotetext{
Recebido em 30/04/2019

Aprovado em 05/05/2020

Conflito de interesses: inexistente

Suporte financeiro: não houve
} 